\title{
TOLERÂNCIA DE CULTIVARES DE SOJA A DOIS NÍVEIS DE ALUMÍNIO EM SOLUÇÕES NUTRITIVAS EM DIFERENTES CONCENTRAÇÕES SALINAS ${ }^{(1)}$
}

HIPólitTo ASSUNÇÃo ANTONIO MASCARENHAS (2), Seção de Leguminosas, CARLOS EDUARDO DE OLIVEIRA CAMARGO (2), Seçâo de Arroz e Cereais de Inverno, e SôNIA MARIA PIERRO FALIVENE, Seção de Leguminosas, Instituto Agronômico.

\section{RESUMO}

Os cultivares Biloxi, Cristalina, Santa-Rosa e UFV-1 foram testados em soluções nutritivas contendo $1 / 10,1 / 5$ e $1 / 2$ da concentraçāo salina, na solução nutritiva completa em presença de 1 e $5 \mathrm{mg}$ de $\mathrm{Al}^{3+} /$ /itro. Os resultados mostraram que na identificaçāo dos cultivares tolerantes dos suscetiveis ao alumínio, tanto poderia ser usada a concentração de sais de $1 / 10$ combinado com $1 \mathrm{mg}$ de Al ${ }^{3+} /$ litro de Al, como $1 / 2$ de concentração de sais combinados com $5 \mathrm{mg}$ de $\mathrm{Al}^{3+} /$ litro e utilizado o comprimento da raiz primária como parâmetro de avaliação. Tanto o peso seco da parte aérea como o đas raízes mostraram tendência de aumentar à medida que cresceu a concentração de sais, independente da concentraçáo de Al3i+ utilizada. Os teores de $\mathrm{Ca}, \mathbf{M g}$ e $\mathrm{K}$ nas partes aéreas das plântulas aumentaram e os de $\mathrm{Al}$ diminuíram à medida que se aumentou a concentração de sais nas soluções. O decréscimo nos teores de Al na parte aérea foi menor para o cultivar Biloxi, independentemente đa concentração de $\mathrm{Al}^{3+}$ utilizada nas soluçōes.

\section{INTRODUÇÃO}

A presença de alumínio trocável ou solúvel em solos ácidos de cerrado tem sido um fator limitante na produtividade das leguminosas

(1) Recebido para publicação a 30 de março de 1983.

(2) Bolsista do CNPq. 
em geral e particularmente na cultura da soja. O efeito da toxicidade de alumínio em soja se caracteriza por uma redução efetiva nas raízes, tornando as plantas indiretamente mais sensiveis à seca e queima pela salinidade (5). Apresenta-se então, como de grande importância para maior produtividade de grão por área, o desenvolvimento de cultivares com tolerância ao aluminio.

A seleção de material tolerante e suscetível ao $\mathrm{Al}^{3+}$ tem sido feita por vários autores, utilizando soluçāo nutritiva com níveis tóxicos deste elemento $(\mathbf{7}, \mathbf{8})$. Foi demonstrado por MASCARENHAS \& CAMARGO (4) que o comprimento da raiz primária foi um parâmetro adequado para a identificação e seleção de cultivares de soja quanto à toxicidade de $\mathrm{Al}^{3+}$ em solução nutritiva. No entanto, CAMARGO (2) em arroz e CAMARGO et alii (3) em trigo demonstraram que a redução do crescimento do sistema radicular pela toxicidade de alumínio poderia ser obtida tanto aumentando sua cuncentração como diminuindo a concentração de sais da solução nutritiva. O objetivo deste trabalho visou verificar se esse fenômeno também ocorreria com soja.

\section{MATERIAL E MẼTODOS}

Foram escolhidas vinte e cinco sementes previamente germinadas dos cultivares Biloxi, Cristalina, Santa Rosa e UFV-1 e adaptadas sobre seis telas de náilon. Cada uma das telas contendo as sementes dos quatro cultivares foi colocada em contato com a soluçáo nutritiva exis. tente em seis vasilhas plásticas de 8,3 litros de capacidade cada uma.

A composição das soluçōes nutritivas foi a seguinte: 1) $1 \mathrm{mg}$ de $\mathrm{Al}^{3+} /$ litro com metade da concentração da solução nutritiva completa; 2) $5 \mathrm{mg}$ de $\mathrm{Al}^{3+} /$ litro com metade da concentração da solução nutritiva completa; 3) $1 \mathrm{mg}$ de $\mathrm{Al}^{3 *} /$ litro com $1 / 5$ da concentração da solução nutritiva completa; 4) $5 \mathrm{mg}$ de $\mathrm{Al}^{3+} /$ litro com $1 / 5$ da concentração da solução nutritiva completa: 5) $1 \mathrm{mg}$ de $\mathrm{Al}^{3+} /$ litro com $1 / 10$ da concentração da solução nutritiva completa; 6) $5 \mathrm{mg}$ de $\mathrm{Al}^{3+} /$ itro com $1 / 10$ da concentração da solução nutritiva completa.

A concentração da solução nutritiva completa desenvolvida por MOORE et alii (6), foi a seguinte: $\mathrm{CA}\left(\mathrm{NO}_{3}\right)_{2}=4 \mathrm{mM} ; \mathrm{MgSO}_{4}=2 \mathrm{mM}$; $\mathrm{KNO}_{3}=4 \mathrm{mM} ;\left(\mathrm{NH}_{4}\right)_{2} \mathrm{SO}_{4}=0,4 \mathrm{mM} ; \mathrm{MnSO}_{4}=2 \mu \mathrm{M} ; \mathrm{CuSO}_{4}=0,3 \mu \mathrm{M}$; $\mathrm{ZnSO}_{4}=0,8 \mu \mathrm{M} ; \mathrm{NaCl}=30 \mu \mathrm{M} ; \mathrm{Na}_{2} \mathrm{MOO}_{4}=0,10_{\mu} \mathrm{M}$ e $\mathrm{H}_{3} \mathrm{BO}_{3}=10_{\mu} \mathrm{M}$.

Nas soluções-tratamento, o fósforo foi omitido parn evitar a possivel precipitação do alumínio como $\mathrm{Al}(\mathrm{OH})_{3}$ e a concentração de ferro foi constante e equivalente a $10 \mu \mathrm{M}$ na forma de $\mathrm{FeCl}_{3}$.

O nível da solução-tratamento das vasilhas plásticas tocava embaixo da tela de náilon de maneira que as sementes foram martidas úmidas e as radículas emergentes tinham um pronto suprimento de nutrientes. O $\mathrm{pH}$ da solução foi previamente ajustado para 4,0 com $\mathrm{H}_{2} \mathrm{SO}_{4} 1 \mathrm{~N}$ man- 
tendo-se próximo deste valor por ajustamentos diários durante todo o experimento. As soluções foram continuamente arejadas $e$, as vasilhas plásticas contendo as soluçōes, colocadas em banho-maria com temperatura de $25 \pm 1^{\circ} \mathrm{C}$ dentro do laboratório. $\mathrm{O}$ experimento foi mantido permanentemente em luz artificial, e as plantas desenvolveram-se nessas condições por sete dias. $\mathrm{O}$ crescimento radicular foi determinado, medindo-se o comprimento em milímetros das raízes de cada plântula ao final desse período, após o que as plântulas de cada cultivar foram separadas em raízes e parte aérea. A seguir, as partes das plântulas de cada tratamento foram colocadas para secar em estufa por cinco dias a $60^{\circ} \mathrm{C}$, para determinação do peso seco em gramas. As partes aéreas foram analisadas quanto aos teores de $\mathrm{K}, \mathrm{Ca}, \mathrm{Mg}$ e $\mathrm{Al}$ segundo a metodologia preconizada por BATAGLIA et alii (1).

O delineamento empregado foi parcelas subdivididas, sendo as parcelas compostas pelas três concentrações de sais, as subparcelas pelas duas concentrações de alumínio e as subsubparcelas pelos quatro cultivares de soja estudados. Foram feitas duas repetiçōes para cadia uma das solúções-tratamentos.

Os dados foram analisados, considerando-se as médias de crescimento da raiz, peso seco das raizes e parte aérea das 25 plântulas de cada cultivar submetidas a crescimento durante sete dias em soluções-tratamento.

\section{RESULTADOS E DISCUSSão}

A análise de variância para comprimento da raiz mostrou que houve significância para cultivares, assim como para concentraçōes de sais e alumínio. Para comprimento de raízes, os dados do quadro 1 mostram que, independente da concentração de alumínio, 1/10 da concentração de sais diferiu de $1 / 5$ ou $1 / 2$ pelo teste de Duncan a $5 \%$. Por outro lado, $5 \mathrm{mg}$ de $\mathrm{Al}^{3+} /$ litro diminuiu bastante o comprimento da raiz em relação a $1 \mathrm{mg}$ de $\mathrm{Al}^{3+} /$ litro, independente da concentração de sais utilizada nas soluções-tratamento (Quadro 1 ).

QUADRO 1. Efeito da concentração de sais e de Alja na solução nutritiva, sobre o comprimento das raízes de soja. Médias de repetiçōes e de cultivares

Concentração de sais

$$
\text { Comprimento das raízes }(\mathrm{mm})(1)
$$

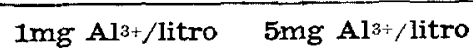

\begin{tabular}{rlr}
71,00 & 19,50 & $90,50 \mathrm{~b}$ \\
149,75 & 31,13 & $180,88 \mathrm{a}$ \\
165,25 & 65,13 & $230,38 \mathrm{a}$ \\
$128,67 \mathrm{a}$ & $\mathbf{3 9 , 5 9 b}$ & \\
\hline
\end{tabular}

(1) Médias seguidas de letras iguais não diferem significativamente, na coluna vertical, pelo teste de Duncan a $\mathbf{5 \%}$ e, na horizontal, pelo teste $\mathrm{t}$ a $\mathbf{5} \%$. 
Pela figura 1, observa-se que tanto ao nível de $1 \mathrm{mg}$ de $\mathrm{Al}^{3+} /$ litro como no de $5 \mathrm{mg}$ de $\mathrm{Al}^{3+} /$ litro, houve aumento do comprimento da raiz à medida que se aumentou a concentração de sais de 1/10 para 1/2, apesar de o comprimento da raiz ser menor a $5 \mathrm{mg}$ de $\mathrm{Al}^{3^{-}} /$litro, por ser esta concentração muito alta para soja, conforme foi relatado por MASCARENHAS et alii (4).

Ao nível de $1 \mathrm{mg}$ de $\mathrm{Al}^{3-} /$ liitro e a $1 / 10$ de concentração de sais (Figura 1 e Quadro 2) o comprimento da raiz do cultivar Cristalina mostrou ser igual ao do Biloxi e Santa-Rosa e maior em relação ao do UFV-1; assim foram confirmados os dados obtidos com os mesmos cultivares em trabalho anterior (4). Tanto em $1 / 5$ como em $1 / 2$ da concentração de sais na solução, o cultivar Cristalina se igualou ac UFV-1 em comprimento da raiz, sendo Santa-Rosa o melhor com $1 / 5$ de concentração de sais na solução-tratamento.

QUADRO 2. Comprimento médio das raizes de quatro cultivares de soja após sete dias de crescimento em soluções nutritivas contendo duas concentrações de alumínio, combinadas com três concentrações salinas

\begin{tabular}{|c|c|c|c|c|c|c|}
\hline \multirow{3}{*}{ Cultivares } & \multicolumn{6}{|c|}{ Comprimento médio de raízes $(\mathrm{mm})$ (1) } \\
\hline & \multicolumn{3}{|c|}{ Concentração de sais } & \multicolumn{3}{|c|}{ Concentração de sais } \\
\hline & $1 / 10$ & $1 / 5$ & $1 / 2$ & $1 / 10$ & $1 / 5$ & $1 / 2$ \\
\hline & \multicolumn{3}{|c|}{ 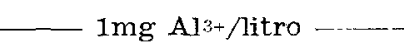 } & $-5 \mathrm{mg}$ & \multicolumn{2}{|c|}{$\mathrm{Al}^{3+} /$ litro -} \\
\hline Biloxi & $122 \mathrm{ab}$ & $291 \mathrm{ab}$ & $357 \mathrm{a}$ & $41 \mathrm{a}$ & $62 \mathrm{a}$ & $132 \mathrm{a}$ \\
\hline Cristalina & $183 a$ & $269 b$ & $328 \mathrm{a}$ & $39 a$ & $66 \mathrm{a}$ & $131 a$ \\
\hline Santa Rosa & $151 a b$ & $358 \mathrm{a}$ & $344 a b$ & $39 a$ & $62 \mathrm{a}$ & $152 a$ \\
\hline UFV-1 & $120 b$ & $280 \mathrm{~b}$ & $293 \mathrm{~b}$ & $37 \mathrm{a}$ & $59 a$ & $106 b$ \\
\hline d.m.s $5 \%$ & 66 & 68 & 57 & 24 & 22 & 23 \\
\hline
\end{tabular}

(i) Teste de Duncan a $5 \%$.

Pela figura 1 e quadro 2 ao nível de $5 \mathrm{mg}$ de $\mathrm{Al}^{3+} /$ litro - tanto a $1 / 10$ como $1 / 5$ da concentração de sais - vê-se que não houve diferença no comprimento de raízes dos cultivares devido à toxicidade causada pela alta concentração de $\mathrm{Al}^{3+}$. Para $1 / 2$ da concentração, nota-se uma nítida separação entre o cultivar UFV-1 dos demais (Figura 2). Para separar os cultivares tolerantes dos sensíveis, os resultados obtidos sugerem que tanto poderia ser usada a concentração de sais de $1 / 10$ combinada com Img ae $\mathrm{Al}^{\mathrm{s}-/}$ /litro ou $1 / 2$ combinada com $5 \mathrm{mg}$ de $\mathrm{Al}^{3+} /$ litro.

Pelo quadro 3, observa-se que tanto o peso seco da parte aérea como o das raízes mostraram tendência de aumentar à medida que se aumentou a concentração de sais, independentemente do $\mathrm{Al}^{3+}$, mas que os pesos foram menores com o tratamento de $5 \mathrm{mg}$ cle $\mathrm{Al}^{3-} /$ litro. 


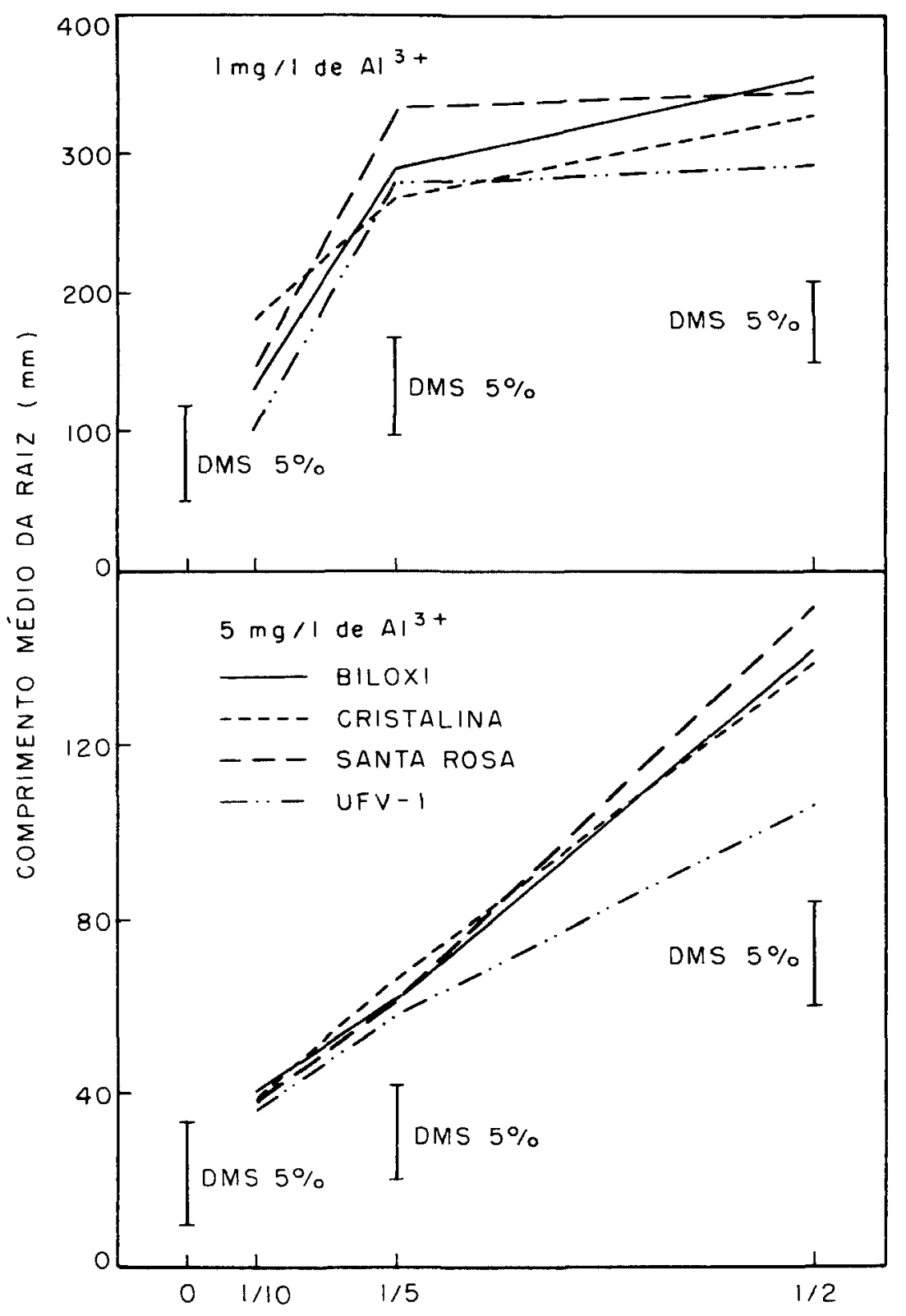

\section{CONCENTRAÇ̃̃O DA SOLUCÃO}

Figura 1. Comprimento médio das raízes de quatro cultivares de soja estudados eñ soluções nutritivas contendo duas diferentes concentrações de Al3+ combinadas com três concentrações salinas. 

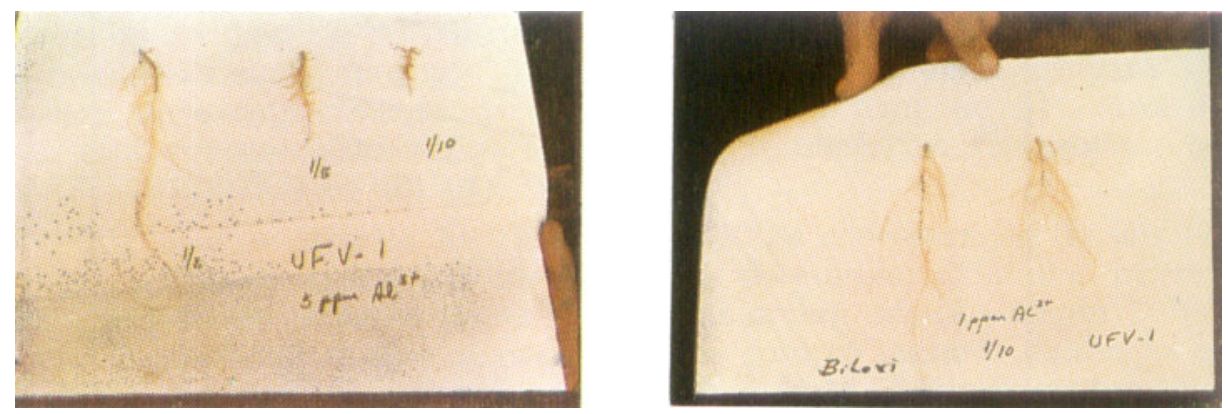

FIGURA 2. Raizes do cultivar UFV-1 após sete dias em soluções nutritivas contendo $5 \mathrm{mg}$ /litro de Al3+, combinadas com três concentrações salinas (esquerda). Raízes dos cultivares Biloxi e UFV-1 após sete dias em solução nutritiva, contendo Img de $\mathrm{Al}^{3+} /$ litro com $1 / 10$ de concentração salina (direita).

QUADRO 3. Peso seco médio da parte aérea e da raiz de quatro cultivares de soja após sete dias de crescimento em soluções nutritivas contendo duas concentrações de alumínio combinadas com três concentrações salinas de solução

\begin{tabular}{|c|c|c|c|c|c|c|}
\hline \multirow{3}{*}{ Cultivares } & \multicolumn{6}{|c|}{ Matéria seca das plantas (mg/planta) } \\
\hline & \multicolumn{3}{|c|}{ Concentração de sais } & \multicolumn{3}{|c|}{ Concentração de sais } \\
\hline & $1 / 10$ & $1 / 5$ & $1 / 2$ & $1 / 10$ & $1 / 5$ & $1 / 2$ \\
\hline & \multicolumn{3}{|c|}{ - $1 \mathrm{mg} \mathrm{Al}{ }^{3+/ \text { litro }}$} & \multicolumn{3}{|c|}{$-5 \mathrm{mg} \mathrm{Al} 3+/$ litro } \\
\hline \multicolumn{7}{|l|}{ Parte aérea } \\
\hline Biloxi & 69 & 77 & 85 & 41 & 62 & 68 \\
\hline Cristalina & 60 & 68 & 72 & 42 & 66 & 61 \\
\hline Santa Rosa & 59 & 71 & 81 & 46 & 59 & 54 \\
\hline UFV-1 & 62 & 78 & 84 & 43 & 70 & 62 \\
\hline \multicolumn{7}{|l|}{ Raizes } \\
\hline Biloxi & 16 & 18 & 21 & 8 & 11 & 15 \\
\hline Cristalina & 18 & 19 & 21 & 9 & 13 & 17 \\
\hline Santa Rosa & 21 & 26 & 26 & 11 & 13 & 19 \\
\hline UEV-1 & 18 & 19 & 20 & 10 & 11 & 18 \\
\hline
\end{tabular}

Os teores de $\mathrm{Ca}, \mathrm{Mg}$ e $\mathrm{K}$ na parte aérea de plântulas tenderam a aumentar à medida que se aumentou a concentração de sais nas soluções e houve um decréscimo nos teores de $\mathrm{Al}$, decréscimo esse menor para $\mathrm{O}$ cultivar Bilóxi para os dois niveis de $\mathrm{Al}^{3+}$, conforme se pode observar no quadro 4. 


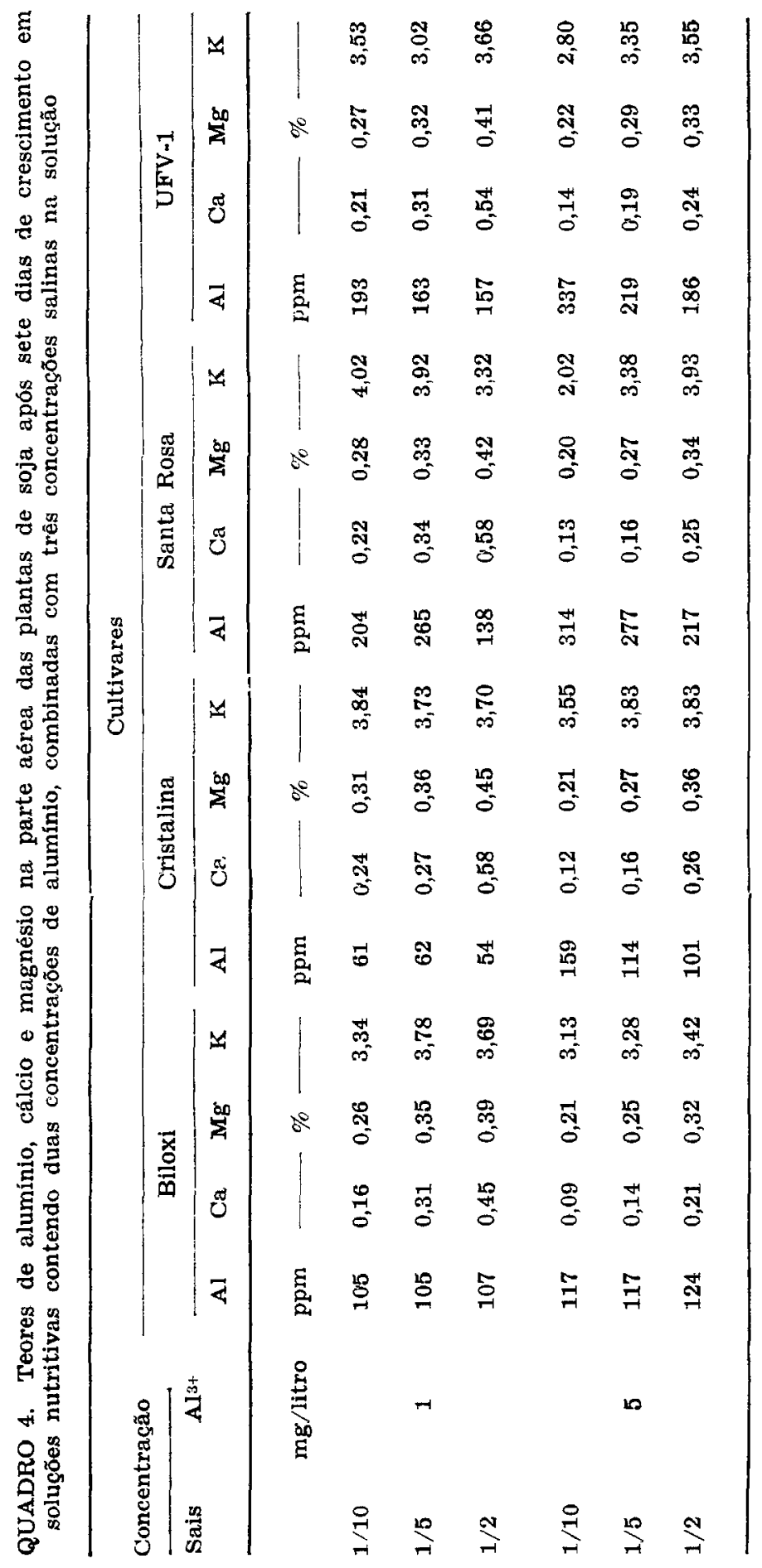

Bragantia, Campinas, 43(2):459-466, 1984 


\section{SUMMARY}

\section{INFLUENCE OF SALT CONCENTRATION IN NUTRIENT SOLUTION ON TOLERANCE TO ALUMINUM TOXICITY IN SOYBEAN CULTIVARS}

The aluminum tolerance of four soybean cultivars was stucied in nutrient solutions using different levels of this element combined with three different salt concentrations. The results showed that to separate the tolerant from the susceptible cultivars at $1 \mathrm{mg}$ Als $3+/$ liter, the salt concentration of $1 / 10$ of the complete nutrient solution was sufficient. The combination of $5 \mathrm{mg} \mathrm{Al}{ }^{3+} / \mathrm{liter}$ with $1 / 2$ salt concentration can also be used considering the root lenght of the primary root as the parameter. The tops and root dry matter weight increased as the salt concentration in solution increased, independent of the Alis concentration. Higher $\mathrm{Ca}, \mathrm{Mg}$ and $\mathrm{K}$ and lower $\mathrm{Al}$ concentrations were observed in the tops as the concentration of the salt in the solution was increased. This decrease in Al concentration was less evident for the cultivar Biloxi at both levels, 1 and $5 \mathrm{mg}$ $\mathrm{Al}^{3+} /$ liter.

\section{REFERENCIAS BIBLIOGRAFICAS}

1. Bataglia, O. C.; Teixeira, J. P. F.; FURLANI, P. R.; FURLANI, A. M. G.; GALLO, J. R. Métodos de análise química de plantas. Campinas, Instituto Agronômico, 1978. 31p. (Circular, 87)

2. CAMARGo, C. E. O. Tolerância de cultivares de arroz a dois niveis de alumínio em soluções nutritivas contendo diferentes concentrações de sais. Bragantia, Campinas, 43(2):381-388, 1984.

3. —— OLIVEIRA, O. F.; LAVORENTI, A. Efeito de diferentes concentrações de sais em solução nutritiva na tolerância de cultivares de trigo a toxicidade de alumínio. Bragantia, Campinas, 40:93-101, 1981.

4. MASCARENHAS, H. A. A.; CAMARGO, C. E. O.; FALTVENE, S. M. P. Efeito do alumínio sobre o crescimento de raízes, peso seco da parte aérea e raiz de diferentes cultivares de soja. Bragantia, Campinas, 43(1):191-200, 1984.

5. - ; MIRANDA, M. A. C. de; BRAGA, N. R.; HIROCE, R.; BULISANI, E. A. Comportamento diferencial de dois cultivares de soja (Glycine max (L.) Merrill) frente à toxicidade de cloro e alumínio. Revista Brasileira de Ciência do Solo, Campinas, 4:121-123, 1980.

6. MOORE, D. P.; KRONSTAD, W. E.; METZGER, R. Screening wheat for aluminum tolerance. In: WORKSHOP ON PLANT ADAPTATIONS TO MINERAL STRESS IN PROBLEM SOILS. Beltsville, Maryland, 1976. Proceedings. p.287-295.

7. SAPRA, V. T.; MEBRAHTO, T.; MUGWIRA, L. M. Soybean germplasm and cultivar aluminum tolerance in nutrient solution and in Braden clay loam soil. Agronomy Journal, 74:687-690, 1982.

8. SARTAIN, J. B. Differential effects of aluminum on tops and root growth, nutrient accumulation and nodulation of several soybean varieties. Universi. dade de Carolina do Norte, 1974. 119p. Tese (Ph.D.) 